\title{
HOMOGENEOUS SUBSETS OF A LIPSCHITZ GRAPH AND THE CORONA THEOREM
}

\author{
Brady Max NewDelman
}

Abstract

This paper proves the Corona Theorem to be affirmative for domains in the complex plane bounded by thick subsets of a Lipschitz graph. Specifically, the boundary of these domains $E_{0}$ has a Carleson lower density:

$$
\Lambda\left(B(z, r) \cap E_{0}\right)>\epsilon_{0} r \quad \text { for all } z \in E_{0}, \quad \text { and all } r>0 .
$$

\section{Introduction}

Let $\mathcal{A}: \mathbb{R} \rightarrow \mathbb{R}$ be an $M$-Lipschitz continuous function. Thus $\mathcal{A}$ has a derivative almost everywhere such that $\left\|\mathcal{A}^{\prime}\right\|_{L^{\infty}}=M$. Let $\Gamma$ be the Lipschitz graph parametrically defined by $z(x)=x+i \mathcal{A}(x)$ in the extended complex plane, and let $E_{0}$ be a closed set contained in $\Gamma$ with

$$
\Lambda\left(B(z, r) \cap E_{0}\right)>\epsilon_{0} r \quad \text { for all } z \in E_{0}, \quad \text { and all } r>0,
$$

where $\Lambda$ is linear measure in the plane, $B(z, r)$ is the open ball about $z$ of radius $r$, and $\epsilon_{0}>0$. The constant $\epsilon_{0}$ is called the Carleson lower density. Any measurable subset of $\Gamma$ with a positive Carleson lower density is called homogeneous in $\Gamma$.

Set $\Omega=\mathbb{C}^{*} \backslash E_{0}$, and let $H^{\infty}(\Omega)$ denote the space of bounded analytic functions on $\Omega$. In this paper, we prove:

Theorem 1.1 (The Corona Theorem). Given $f_{1}, \ldots, f_{n} \in H^{\infty}(\Omega)$ and $\mu>0$ with the property that $\mu \leq \max \left\{\left|f_{j}(z)\right|: 1 \leq j \leq n\right\} \leq 1$ for every $z \in \Omega$, there exist $g_{1}, \ldots, g_{n} \in H^{\infty}(\Omega)$ such that $f_{1} g_{1}+\cdots+f_{n} g_{n} \equiv 1$ on $\Omega$.

We will refer to the functions $\left\{f_{j}\right\}_{j=1}^{n}$ and $\left\{g_{j}\right\}_{j=1}^{n}$ as the corona data and corona solutions respectively, and we will refer to $\mu$ and $n$ as the corona constants.

2000 Mathematics Subject Classification. 30-XX.

Key words. Corona, Harmonic, Measure, Homogeneous, Lipschitz. 
There is an alternative way of viewing the theorem in the language of uniform algebras. Let us denote by $\mathcal{M}=\mathcal{M}\left(H^{\infty}(\Omega)\right)$ the maximum ideal space of $H^{\infty}(\Omega)$. When $H^{\infty}(\Omega)$ separates the points of $\Omega$, we can identify elements of $\Omega$ with pointwise evaluation functionals in $\mathcal{M}$. Under this identification, the theorem becomes equivalent to determining whether $\Omega$ is dense in $\mathcal{M}$ in the Gelfand topology. It is in this context where the theorem gets its name; whereby, in the special case where $\Omega$ is the unit disk, $\mathbb{D}$, we can think of $\mathbb{D}$ as being the sun, and $\mathcal{M} \backslash \overline{\mathbb{D}}$ as being the sun's corona.

Lennart Carleson (1962) proved the first corona theorem for the case of the disk [4]. His proof was subsequently simplified (using a $\bar{\partial}$ equation) by Hörmander [15], and later by a clever proof by Wolff ([8], [10]). The theorem was swiftly adapted to the case of finitely connected domains (Alling [1], $[\mathbf{2}]$; Stout [24], [25], [26]; and others $[\mathbf{6}],[\mathbf{7}],[\mathbf{2 3}]$ ). Each proof gave new insight into the structure of $H^{\infty}$. The finitely connected domain proofs were fundamentally based upon admixing localized corona solutions for overlapping simply connected components. One major drawback to this method was that the bounds of the corona solutions, $\left\|g_{j}\right\|_{\infty}$, were dependent on the number of boundary components. This was an unfortunate hindrance as any planar domain can be exhausted by a sequence of finitely connected domains. Without a uniform bound on the corona solutions for the approximating domains, any method of taking normal limits was futile. In that direction, Gamelin [9] observed that the corona theorem for all planar domains would be true if and only if there existed a uniform bound for finitely connected domains which is independent of the number of boundary components. A proof to the corona problem for all planar domains remained a mystery.

Further investigations into the corona problem revealed a connection between interpolating sequences, boundary thickness, and the Cauchy transform. Along those lines, Carleson made another breakthrough by proving the corona theorem for domains with homogeneous boundary contained in the real line (homogeneous Denjoy domains). The significance of his result was that these domains are infinitely connected. Carleson lifted the corona data to the universal covering surface (where the corona solutions exist) and then mapped the solutions back to the original domain by an explicit projection operator invented by Forelli [7]. This concept was later simplified by Jones and Marshall [19]. They determined that if the critical points of the Green's function for a domain form an interpolating sequence, then there exists a projection operator and the corona theorem is affirmative. Moreover, they gave conditions 
necessary for determining when the critical points are indeed an interpolating sequence; one such condition can easily be proved when the boundary is homogeneous. Following these results, the corona problem for all planar domains bounded by a homogeneous subset of a graph seemed promising.

Peter Jones was the first to propose the idea of the corona problem for domains whose boundary lies in a Lipschitz graph [18]. He was motivated by the Denjoy conjecture, a consequence of Calderón's theorem on Cauchy integrals, which suggested that the space of bounded analytic functions was significantly abundant for these domains. Thereby, one might be able to construct "by hand" the corona solutions. As mentioned by Jones, the difficulty in the Lipschitz case was the lack of symmetry. At that time, the deepest results for the corona theorem were in the Denjoy domains $(\Omega=\bar{\Omega})$ as in $[\mathbf{5}],[\mathbf{1 9}]$, and [11]. These proofs made explicit use of the symmetry of the domains, either by confining the critical points to real intervals or by creating analytic functions by means of Schwarz reflection. Nonetheless, Jones (unpublished) proved the corona theorem for domains bounded by a homogeneous subset of a Lipschitz graph. He constructed by hand a projection operator akin to Forelli's.

For our proof, we work directly on the underlying space $\Omega$ without localizing the critical points of the Green's function, which can be cumbersome. We divide $\Omega$ into two overlapping simply connected regions, $\widetilde{\Omega}^{+}$ and $\widetilde{\Omega}^{-}$. On each region, we use Carleson's simply connected result to obtain regional corona solutions, $\left\{g_{j}^{+}\right\}_{j=1}^{n}$ and $\left\{g_{j}^{-}\right\}_{j=1}^{n}$. Starting in $\widetilde{\Omega}^{+}$, we constructively solve a particular $\bar{\partial}$ equation to modify $\left\{g_{j}^{+}\right\}_{j=1}^{n}$ so that $\max _{j}\left|g_{j}^{+}(z)-g_{j}^{-}(z)\right|$ is reduced in the overlap of the regions. After the modification, we do a similar procedure in $\widetilde{\Omega}^{-}$to reduce the differences even more, then iterate the procedure. The result of the iteration gets us two uniformly bounded sequences of solutions on each region. The $\bar{\partial}$ equation was constructed specifically so that the normal limits of the sequences agree on the overlap of the regions.

In proving the theorem, we assume that $E_{0}$ consists of a finite union of closed intervals in $\Gamma$, two of which are unbounded. This assumption is easily removed by a normal families argument provided that the number of intervals does not control the bounds of the corona solutions. ${ }^{1}$ To be clear, when we use the phrase, " $J$ is an interval in $\Gamma$ " we mean $p(J)$ is an

\footnotetext{
${ }^{1}$ The homogeneous condition combined with the fact that $E_{0}$ is closed implies that $\epsilon_{0} \leq 1 / 2$. The reason being that a complementary open interval $F \subset \Gamma \backslash E_{0}$ is not empty. As such, the double of $F$ has a density less than $1 / 2$.
} 
interval in $\mathbb{R}$ for the projection $p: \Gamma \rightarrow \mathbb{R}$ defined by $p(z(x))=x$. It will also be convenient for us to consider the Lipschitz angle $\alpha=\tan ^{-1}(M)$ for most of our calculations, instead of the slope $M$.

We mention here that there are two conditions equivalent to (1.1):

Lemma 1.2. When $\Gamma$ is an $M$-Lipschitz graph and $E_{0} \subset \Gamma$, the following three conditions are equivalent:

i) $E_{0}$ is homogeneous with a Carleson lower density $\epsilon_{0}$.

ii) There exists an $\epsilon_{1}>0$ such that $\left|p\left(E_{0}\right) \cap(x-r, x+r)\right|>\epsilon_{1} r$ for all $z=x+i y \in E_{0}$, and all $r>0$.

iii) There exists an $\epsilon_{2}>0$ such that if we denote by $J_{z, r}=J_{L} \cup J_{R}$ the interval in $\Gamma$ containing $z ; J_{L}$ is the subinterval having $z$ as a right endpoint, and $J_{R}$ is the subinterval having $z$ as a left endpoint with $\Lambda\left(J_{L}\right)=\Lambda\left(J_{R}\right)=r$, then $\Lambda\left(J_{z, r} \cap E_{0}\right)>\epsilon_{2} r$, for all $z \in E_{0}$ and all $r>0$.

In addition, if either $i)$, ii), or iii) hold, then

iv) There exists an $\epsilon_{3}>0$ such that cap $\left(B(z, r) \cap E_{0}\right)>\epsilon_{3} r$ for all $z \in$ $E_{0}$, and all $r>0$.

The third item, iii), has the advantage that it applies to more general curves, while iv) is even more general: it says $E_{0}$ is uniformly perfect (see [22]). The crux of the proof for Lemma 1.2 is based upon the relationship of the projected length:

$$
\Lambda(J) \geq|p(J)| \geq \cos (\alpha) \Lambda(J) \quad \text { for an interval } J \subset \Gamma .
$$

Proof of Lemma 1.2: Let us first assume that i) holds. Fix $z=x+i y \in$ $\Gamma$ and $r>0$. Since the projected mass of $B(z, r) \cap E_{0}$ lies inside of $(x-r, x+r) \cap p\left(E_{0}\right)$ and

$$
\left|p\left(B(z, r) \cap E_{0}\right)\right| \geq \cos (\alpha) \Lambda\left(B(z, r) \cap E_{0}\right)>\cos (\alpha) \epsilon_{0} r,
$$

condition ii) holds with $\epsilon_{1}=\cos (\alpha) \epsilon_{0}$.

Now assume that ii) holds, and fix $z \in \Gamma$ and $r>0$. By simple geometric considerations, we see that $B(z, r \cos (\alpha)) \cap \Gamma \subset J_{z, r}$. This implies

$$
\begin{aligned}
\Lambda\left(J_{z, r} \cap E_{0}\right) & \geq \Lambda\left(B(z, r \cos (\alpha)) \cap E_{0}\right) \\
& \geq\left|(x-r \cos (\alpha), x+r \cos (\alpha)) \cap p\left(E_{0}\right)\right|>\cos (\alpha) \epsilon_{1} r .
\end{aligned}
$$

The last inequality is from ii). This implies condition iii) with $\epsilon_{2}=$ $\cos (\alpha) \epsilon_{1}$. 
Showing that iii) implies i) is simple as we can make the interval $J_{z, r}$ inside the ball $B(z, r)$. Then condition iii) implies $\Lambda\left(B(z, r) \cap E_{0}\right)>\epsilon_{2} r$. Thus $E_{0}$ is homogeneous with a Carleson lower density $\epsilon_{2}$.

Lastly, from the proof of Theorem III.11 in [27], we have the relationship for $E_{0} \subset \Gamma$,

$$
\operatorname{cap}\left(B(z, r) \cap E_{0}\right) \geq \frac{\cos (\alpha) \Lambda\left(B(z, r) \cap E_{0}\right)}{2 e} .
$$

This tells us that i) implies iv) with $\epsilon_{3}=\frac{\epsilon_{0} \cos (\alpha)}{2 e}$.

For the proof of Theorem 1.1, we make the additional assumption that the tangent to $\Gamma$ at a point $\zeta \in \Gamma$ is constant whenever $\zeta \in \Gamma \backslash E_{0}$. This comes without any loss of generality. Specifically, if we write $\Gamma \backslash E_{0}=$ $\cup_{k} F_{k}$, then we define (see Figure 1)

$$
c_{k}=\tan ^{-1}\left[\mathcal{A}^{\prime}(x)\right], \quad \text { when } z=x+i y \in F_{k} .
$$

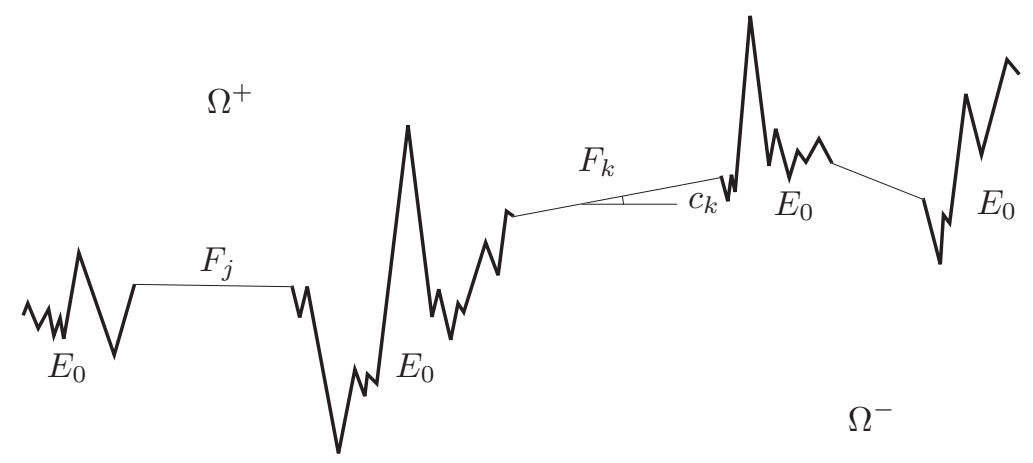

Figure 1. The open interval $F_{k}$ makes an angle $c_{k}$ with the $x$-axis.

Let us now fix some notation that will be used throughout the whole paper. We define a tent region over an interval $J=\left(z_{1}, z_{2}\right)$ with acute angle $\gamma$ by

$$
T_{(J, \gamma)}=\left\{z: 0<\arg \left[\frac{z-z_{1}}{z_{2}-z_{1}}\right]<\gamma \quad \text { and } \quad 0<\arg \left[\frac{z_{1}-z_{2}}{z-z_{2}}\right]<\gamma\right\} .
$$


Notice that $\Gamma$ divides the plane into two simply connected components, $\Omega^{+}$and $\Omega^{-}$, where $\Omega^{+}$lies above $\Gamma$ and $\Omega^{-}$lies below $\Gamma$. With these components, we fix two conformal maps and their inverses:

$$
\Phi^{+}(z): \mathbb{H}^{+} \rightarrow \Omega^{+}, \quad \Psi^{+}(z)=\left(\Phi^{+}(z)\right)^{-1}: \Omega^{+} \rightarrow \mathbb{H}^{+},
$$

and

$$
\Phi^{-}(z): \mathbb{H}^{-} \rightarrow \Omega^{-}, \quad \Psi^{-}(z)=\left(\Phi^{-}(z)\right)^{-1}: \Omega^{-} \rightarrow \mathbb{H}^{-}
$$

We ask that $\Phi^{+}(\infty)=\infty$ and $\Phi^{-}(\infty)=\infty$. From Carathéodory's theorem we can extend our maps to homeomorphisms so that $\Phi^{+}: \overline{\mathbb{H}^{+}} \rightarrow$ $\overline{\Omega^{+}}$and $\Phi^{-}: \overline{\mathbb{H}^{-}} \rightarrow \overline{\Omega^{-}}$respectively (see [20, Theorem I.1]). ${ }^{2}$ We will be using two facts about about $\Phi^{+}$and $\Phi^{-}$:

Lemma 1.3. The closed set $E^{+}=\Psi^{+}\left(E_{0}\right)$ has a Carleson lower density $\epsilon=\epsilon\left(\epsilon_{0}, \alpha\right)$ in $\mathbb{R}$. Likewise, $E^{-}=\Psi^{-}\left(E_{0}\right)$ has a Carleson lower density $\epsilon=\epsilon\left(\epsilon_{0}, \alpha\right)$ in $\mathbb{R}$.

Lemma 1.4. For any interval $F_{j} \subset \Gamma \backslash E_{0}$,

$$
\Phi^{+}\left(T_{\left(\Psi+\left(F_{j}\right), \gamma\right)}\right) \subset T_{\left(F_{j}, 3 \gamma\right)} \quad \text { for } \gamma<\pi / 12 .
$$

Likewise,

$$
\Phi^{-}\left(\overline{T_{\left(\Psi^{-}\left(F_{j}\right), \gamma\right)}}\right) \subset\left(T_{\left(F_{j}, 3 \gamma\right)}\right)^{*} \quad \text { for } \gamma<\pi / 12,
$$

where $*$ denotes reflection across $F_{j}$.

Lemma 1.3 tells us that homogeneity is preserved by the maps (although the Carleson lower densities may be different), while Lemma 1.4 tells us that obtuse tents are mapped into obtuse tents. It should be mentioned that $\pi / 12$ is not crucial for Lemma 1.4. We made this choice since the acute angle gets tripled in the lemma and, throughout this paper, we will only consider tents that have an acute angle less than $\pi / 4$.

Proof of Lemma 1.3: We use a result of Kenig [20]: if $\nu$ is the measure on $\mathbb{R}$ whose density is $\left|\left(\Phi^{+}\right)^{\prime}(x)\right|$, then $\nu \in \mathcal{A}_{2}$ on $\mathbb{R}$, where $\mathcal{A}_{2}$ is the class of Muckenhoupt. Now fix $r>0, x \in E^{+}, z=\Phi^{+}(x)$ and have $I=(x-r, x+r)$. Write $I=I_{L} \cup I_{R}$, where $I_{L}=(x-r, x]$ and $I_{R}=[x, x+r)$ and denote $K=\Phi^{+}(I), K_{L}=\Phi^{+}\left(I_{L}\right)$, and $K_{R}=\Phi^{+}\left(I_{R}\right)$.

${ }^{2}$ By placing suitable minus signs, we may assume that $\mathfrak{R e}\left\{\Psi^{ \pm}\left(z_{1}\right)\right\}<\mathfrak{R e}\left\{\Psi^{ \pm}\left(z_{2}\right)\right\}$ whenever $z_{1}, z_{2} \in \Gamma$ and $\mathfrak{R e}\left\{z_{1}\right\}<\mathfrak{R e}\left\{z_{2}\right\}$. 
Without loss of generality, let us assume that $\Lambda\left(K_{R}\right) \leq \Lambda\left(K_{L}\right)$. The $\mathcal{A}_{2}$ relationship gives us a lower bound for $\Lambda\left(K_{R}\right)$,

$$
\Lambda\left(K_{R}\right) \geq \frac{1}{C_{2}}\left(\frac{1}{2}\right)^{2} \Lambda(K)
$$

where $C_{2}$ is the $\mathcal{A}_{2}$ constant.

Let $J_{z}$ be the interval inside $K$, as defined as in Lemma 1.2, with $J_{z}=J_{L} \cup J_{R}$, where $J_{R}=K_{R}$ and $J_{L}$ is the interval with right endpoint $z$ and length equal to $\Lambda\left(K_{R}\right)$. From the proof of Lemma 1.2, we know that $\Lambda\left(E_{0} \cap J_{z}\right)>\epsilon_{0} \cos (\alpha) \Lambda\left(K_{R}\right)$, and when we combine this inequality with (1.2) we have

$$
\frac{\Lambda\left(E_{0} \cap K\right)}{\Lambda(K)} \geq \frac{\Lambda\left(E_{0} \cap J_{z}\right)}{\Lambda(K)}>\epsilon_{0} \cos (\alpha) \frac{1}{C_{2}}\left(\frac{1}{2}\right)^{2}
$$

By a result of Muckenhoupt $[\mathbf{2 1}], \nu \in \mathcal{A}_{2}$ on $\mathbb{R}$ implies $\nu \in \mathcal{A}_{\infty}$ on $\mathbb{R}$. Hence, there exist constants $c_{1}>0$ and $c_{2}>0$ independent of $E^{+}$and $r$ such that,

$$
\begin{aligned}
\left|\frac{E^{+} \cap(x-r, x+r)}{(x-r, x+r)}\right| & \geq c_{1}\left(\frac{\Lambda\left(\Phi^{+}\left(E^{+} \cap(x-r, x+r)\right)\right)}{\Lambda\left(\Phi^{+}((x-r, x+r))\right)}\right)^{c_{2}} \\
& =c_{1}\left(\frac{\Lambda\left(E_{0} \cap K\right)}{\Lambda(K)}\right)^{c_{2}}
\end{aligned}
$$

Combining the above relationship with (1.3), we see that $E^{+}$is homogeneous with a Carleson lower density depending only upon $\epsilon_{0}$ and $\alpha$.

Proof of Lemma 1.4: The appearance of the $*$ and the conjugation bar for the statement in the lower half plane arise since the tents have an orientation to be above the intervals. It is not difficult to see that the two statements remain alike upon modifying the arguments in the definition of the tents, and we will only prove the result for the upper half plane.

Fix a tent domain $T_{\left(I_{j}^{+}, \gamma\right)}$ over $I_{j}^{+}=\Psi^{+}\left(F_{j}\right) \in \mathbb{R}$ and write $\log \left[\left(\Phi^{+}\right)^{\prime}\right](z)=$ $f_{1}(z)+i f_{2}(z)$ (take a principle determination). Again from [20], we have a bounded argument for the derivative, that is $\left|f_{2}(z)\right| \leq \alpha$ for all $z \in \mathbb{H}$. As such, we can represent $f_{2}(z)$ with a Poisson integral of the values 
coming from its non-tangential limits on the real line:

$$
\begin{aligned}
f_{2}(z)-c_{j} & =\int_{\mathbb{R}}\left(f_{2}(t)-c_{j}\right) P_{z}(t) d t \\
& =\int_{I_{j}^{+}}\left(f_{2}(t)-c_{j}\right) P_{z}(t) d t+\int_{\mathbb{R} \backslash I_{j}^{+}}\left(f_{2}(t)-c_{j}\right) P_{z}(t) d t \\
& =\int_{\mathbb{R} \backslash I_{j}^{+}}\left(f_{2}(t)-c_{j}\right) P_{z}(t) d t .
\end{aligned}
$$

The final equality holds since $f_{2}=c_{j}$ over $I_{j}^{+}$. Taking absolute values of the above equality we get $\left|f_{2}(z)-c_{j}\right| \leq 2 \alpha\left(1-\omega\left(z, I_{j}^{+}, \mathbb{H}^{+}\right)\right)$. Additionally, if $z \in T_{\left(I_{j}^{+}, \gamma\right)}$ and $\gamma \leq \pi / 12$, then $\left|f_{2}(z)-c_{j}\right| \leq 4 \alpha \gamma / \pi$ by taking simple estimates for harmonic measure. This lets us conclude that the values of the derivative lie in the cone domain:

$$
c_{j}-\frac{4 \alpha \gamma}{\pi} \leq \arg \left[\Phi^{+}(z)^{\prime}\right] \leq c_{j}+\frac{4 \alpha \gamma}{\pi} \quad \text { for } z \in T_{\left(I_{j}^{+}, \gamma\right)}
$$

So that if we denote $I_{j}^{+}=\left(x_{1}, x_{2}\right)$, then

$$
\begin{aligned}
\arg \left[\frac{\Phi^{+}(z)-\Phi^{+}\left(x_{1}\right)}{\Phi^{+}\left(x_{2}\right)-\Phi^{+}\left(x_{1}\right)}\right] & =\arg \left[\frac{\int_{\left[x_{1}, z\right]} \Phi^{\prime}(w) d w}{\Phi^{+}\left(x_{2}\right)-\Phi^{+}\left(x_{1}\right)}\right] \\
& <\left(\gamma+\left(c_{j}+\frac{4 \alpha \gamma}{\pi}\right)\right)-c_{j}<3 \gamma
\end{aligned}
$$

and

$$
\begin{aligned}
\arg \left[\frac{\Phi^{+}\left(x_{1}\right)-\Phi^{+}\left(x_{2}\right)}{\Phi^{+}(z)-\Phi^{+}\left(x_{2}\right)}\right] & =\arg \left[\frac{\Phi^{+}\left(x_{1}\right)-\Phi^{+}\left(x_{2}\right)}{\int_{\left[x_{2}, z\right]} \Phi^{\prime}(w) d w}\right] \\
& <\left(\pi+c_{j}\right)-\left((\pi-\gamma)+\left(c_{j}-\frac{4 \gamma \alpha}{\pi}\right)\right)<3 \gamma
\end{aligned}
$$

We conclude that $\Phi^{+}(z)$ lies in $T_{\left(F_{j}, 3 \gamma\right)}$.

\section{Four crosscuts}

Recall $\Gamma \backslash E_{0}=\cup_{k} F_{k}$, now let $\alpha_{M}=(\pi / 2-\alpha) / 4$, and let $D_{j}^{+}=$ $T_{\left(F_{j}, \alpha_{M}\right)}$ be the tent domain in $\Omega^{+}$over $F_{j}$ with acute angle $\alpha_{M}$, likewise define $D_{j}^{-} \subset \Omega^{-}$. Merging the two tents together for all $j$, we make the diamonds $D_{j}=D_{j}^{+} \cup D_{j}^{-}$. The parameters for $\alpha_{M}$ were chosen so that $\alpha_{M}<\pi / 4$ and $D_{j} \cap D_{k}=\emptyset$ for $j \neq k$ (see Figure 2). 


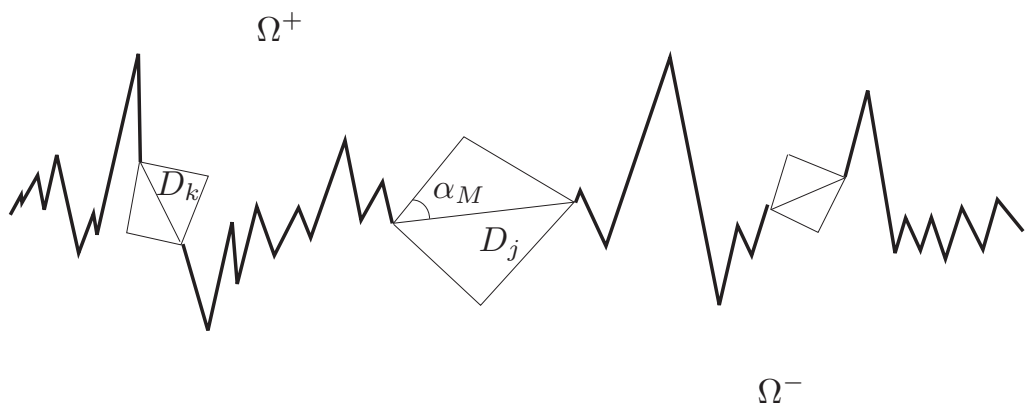

Figure 2. The diamond $D_{j}$ makes an acute angle $\alpha_{M}$ with the open interval $F_{j}$. The angle $\alpha_{M}$ is small enough to ensure that all diamonds are disjoint.

In this section, we construct four families of crosscuts that encompass the open intervals $F_{j}$ and lie inside $D_{j}$. To do so, we will first need some elementary harmonic measure estimates.

In the upper half plane $\mathbb{H}^{+}$,

$$
\omega\left(z, E^{+}, \mathbb{H}^{+}\right)>\epsilon \quad \text { whenever } \quad z=x+i y, \quad \text { and } \quad x \in E^{+} .
$$

This is shown by decomposing the Poisson kernel for the upper half plane into a sum of box kernels centered around $x \in E^{+}\left(P_{z}(t)=\right.$ $\left.\sum_{k} a_{k} \chi_{A_{k}}(t)\right)$. With this representation,

$$
\omega\left(z, E^{+}, \mathbb{H}^{+}\right)=\int_{E^{+}} P_{z}(t) d t=\sum_{k} a_{k}\left|A_{k} \cap E^{+}\right|>\sum_{k} a_{k}\left|A_{k}\right| \epsilon=\epsilon .
$$

If we write the complement of $E^{+}$in the real line as $\bigcup_{j} I_{j}^{+}=\mathbb{R} \backslash E^{+}=$ $\bigcup_{j} \Psi^{+}\left(F_{j}\right)$, then $\omega\left(z, \cup I_{j}^{+}, \mathbb{H}^{+}\right)<1-\epsilon$ when $\mathfrak{R e}\{z\} \in E^{+}$. This tells us that we have the bounds $\omega\left(z, \cup I_{j}^{+}, \mathbb{H}^{+}\right)<1-\epsilon$ on the sides of the vertical half strip defined with base $I_{j}^{+}$extending vertically in the upper half plane. We can apply these bounds to get harmonic measure estimates on the boundaries of the diamonds:

Lemma 2.1. If $z \in \bigcup_{j} \partial T_{\left(I_{j}^{+}, \gamma\right)}$ and $\gamma<\pi / 4$, then $\omega\left(z, \cup I_{j}^{+}, \mathbb{H}^{+}\right)<$ $1-\frac{\epsilon \gamma}{\pi}$.

Proof of Lemma 2.1: Fix $z \in \partial T_{\left(I_{k}^{+}, \gamma\right)}$ for some $k$ and normalize $I_{k}^{+}$ into $(-\pi / 2, \pi / 2)$, and let us denote the half strip over $I_{k}^{+}$by $S^{+}=$ 
$\{x+i y:-\pi / 2<x<\pi / 2: y>0\}$. From the preceding remarks,

$$
\omega\left(z, \cup I_{k}^{+}, \mathbb{H}^{+}\right)<(1-\epsilon)+\epsilon \omega\left(z,[-\pi / 2, \pi / 2], S^{+}\right) .
$$

If we write $z=-\pi / 2+t e^{i \gamma}$, then

$$
\begin{aligned}
\arg [\sin (z)-\sin (-\pi / 2)] & =\arg \left[\int_{[-\pi / 2, z]} \frac{d(\sin (w))}{d w} d w\right] \\
& =\arg \left[\int_{[-\pi / 2, z]} \cos (w) d w\right] \\
& =\arg \left[\int_{0}^{t} \cos \left(-\pi / 2+s e^{i \gamma}\right) e^{i \gamma} d s\right] \\
& =\gamma+\arg \left[\int_{0}^{t} \sin \left(s e^{i \gamma}\right) d s\right]>\gamma .
\end{aligned}
$$

By symmetry, this tells us that $\sin (z) \notin T_{([-1,1], \gamma)}$ when $z \in \partial T_{\left(I_{k}^{+}, \gamma\right)}$, so that

$$
\omega\left(z,[-\pi / 2, \pi / 2], S^{+}\right)=\omega\left(\sin (z),[-1,1], \mathbb{H}^{+}\right)<1-\frac{\gamma}{\pi} .
$$

Hence,

$$
\omega\left(z, \cup I_{k}^{+}, \mathbb{H}^{+}\right)<(1-\epsilon)+\epsilon\left(1-\frac{\gamma}{\pi}\right)=1-\frac{\epsilon \gamma}{\pi} .
$$

We remark that Lemma 2.1 can easily be proved without conformal maps but with a weaker bound on harmonic measure. This comes from the observation that if we denote by $d=\operatorname{dist}\left(z, E^{+}\right)$, then $\mid E^{+} \cap$ $B(z, 2 d) \mid>\epsilon d$. This implies for each $z \in \bigcup_{j} \partial T_{\left(I_{j}^{+}, \gamma\right)}$ there exists a subset of $E^{+}$with linear measure proportionate to the distance of $z$ and the real axis. The upper bounds for harmonic measure now follow from estimating the Poisson kernel over these sets.

With the estimates following from Lemma 2.1, we can now define our desired crosscuts. If we let $\beta_{1}=1-\frac{\epsilon \alpha_{M}}{3 \pi}$ and $\beta_{2}=1-\frac{1}{2} \frac{\epsilon \alpha_{M}}{3 \pi}$, then from Lemma 1.4 and Lemma 2.1,

$$
\gamma_{1}^{+}=\Phi^{+}\left(\left\{z: \omega\left(z, \cup I_{j}^{+}, \mathbb{H}^{+}\right)=\beta_{1}\right\}\right)=\Phi^{+}\left(\delta_{1}^{+}\right) \subset D_{j},
$$

and

$$
\gamma_{2}^{+}=\Phi^{+}\left(\left\{z: \omega\left(z, \cup I_{j}^{+}, \mathbb{H}^{+}\right)=\beta_{2}\right\}\right)=\Phi^{+}\left(\delta_{2}^{+}\right) \subset D_{j} .
$$


Similarly, we define the $\gamma_{1}^{-}, \gamma_{2}^{-}, \delta_{1}^{-}$, and $\delta_{2}^{-}$for the lower half planes. These will be our collection of crosscuts.

Recall, a Carleson contour in the upper half plane is a countable union $\mathcal{C}$ of rectifiable arcs in $\mathbb{H}^{+}$such that for every interval $I \subset \mathbb{R}$,

$$
\Lambda(\mathcal{C} \cap(I \times(0,|I|))) \leq C(\mathcal{C})|I| .
$$

This implies arc length on $\mathcal{C}$ is a Carleson measure with constant $C(\mathcal{C})$.

Lemma 2.2. The crosscuts $\delta_{1}^{+}=\left\{z: \omega\left(z, \cup I_{j}^{+}, \mathbb{H}^{+}\right)=\beta_{1}\right\}$ form a Carleson contour in $\mathbb{H}^{+}$. Likewise, $\delta_{1}^{-}=\left\{z: \omega\left(z, \cup I_{j}^{-}, \mathbb{H}^{-}\right)=\beta_{1}\right\}$ form a Carleson contour in $\mathbb{H}^{-}$.

Proof of Lemma 2.2: First we recall that $\delta_{1}^{+}=\left\{z: \omega\left(z, E^{+}, \mathbb{H}^{+}\right)=1-\right.$ $\left.\beta_{1}\right\}$ lies under the tents $\bigcup_{k} T_{\left(I_{k}, \frac{\alpha_{M}}{3}\right)}$. Next, if we write $E^{+}=\bigcup_{j}\left[a_{j}, b_{j}\right]$, then $^{3}$

$$
\omega\left(z, E^{+}, \mathbb{H}^{+}\right)=\frac{1}{\pi} \sum_{j} \arg \left[\frac{b_{j}-z}{a_{j}-z}\right],
$$

and by taking a derivative,

$$
\omega_{y}(z)+i \omega_{x}(z)=\frac{1}{\pi} \sum_{j}\left(\frac{1}{z-b_{j}}-\frac{1}{z-a_{j}}\right) .
$$

Separating the real and imaginary parts gives us the ratio

$$
\frac{\omega_{x}(z)}{\omega_{y}(z)}=\frac{\sum \frac{\left(b_{j}-a_{j}\right) \mathfrak{I m}\left\{\overline{\left(z-b_{j}\right)\left(z-a_{j}\right)}\right\}}{\left|z-a_{j}\right|^{2}\left|z-b_{j}\right|^{2}}}{\sum \frac{\left(b_{j}-a_{j}\right) \mathfrak{R e}\left\{\overline{\left(z-b_{j}\right)\left(z-a_{j}\right)}\right\}}{\left|z-a_{j}\right|^{2}\left|z-b_{j}\right|^{2}}} .
$$

Suppose $z \in \bigcup_{k} T_{\left(I_{k}, \frac{\alpha_{M}}{3}\right)}$, then $\left|\arg \left[\left(z-b_{j}\right)\left(z-a_{j}\right)\right]\right|<2 \alpha_{M} / 3$ for all $j$; and since $\frac{2 \alpha_{M}}{3}<\frac{\pi}{4}$, this makes

$$
\frac{\left|\mathfrak{I m}\left\{\left(z-b_{j}\right)\left(z-a_{j}\right)\right\}\right|}{\mathfrak{R e}\left\{\left(z-b_{j}\right)\left(z-a_{j}\right)\right\}} \leq \tan \left(\frac{2 \alpha_{M}}{3}\right) \text { for all } j .
$$

It is also clear that $\omega_{y}(z)>0$ for all $z$ in the tents, so that by comparing the like terms in the sums of (2.1) with the ratio in (2.2),

$$
\left|\frac{\omega_{x}(z)}{\omega_{y}(z)}\right| \leq \tan \left(\frac{2 \alpha_{M}}{3}\right)
$$

${ }^{3}$ We use the convention $\arg [\infty, z]=0$ and $\arg [-\infty, z]=\pi$ respectively when $\left[a_{j}, b_{j}\right]=$ $\left[a_{j}, \infty\right]$ and $\left[a_{j}, b_{j}\right]=\left[-\infty, b_{j}\right]$. 
As the curve $\delta_{1}^{+}$is a level set, the gradient of $\omega$ at any point is perpendicular to the tangent of the curve. With the above ratio, we conclude that the tangent to the level curves is bounded in argument by $\frac{2 \alpha_{M}}{3}$. This means that $\delta_{1}^{+}$is a Carleson curve with a constant of $\sec \left(\frac{2 \alpha_{M}}{3}\right)$.

\section{The regions $\mathcal{D}^{+}$and $\mathcal{D}^{-}$}

Now that we have the cross cuts $\left\{\gamma_{j}^{ \pm}\right\}_{j=1,2}$, we may define the following extended domains: let $\widetilde{\Omega}^{+}$be the simply connected domain containing $\Omega^{+}$that is bounded by the closed intervals of $E_{0}$ and the bottom crosscuts $\gamma_{1}^{-}$. As $\Psi^{+}(z)$ has a constant argument on each $F_{j}$, and the crosscuts of $\gamma_{1}^{-}$lie in disjoint diamonds, $\Psi^{+}(z)$ can be extended (by reflecting across each $F_{j}$ ) to a map $\widetilde{\Psi}^{+}: \widetilde{\Omega}^{+} \rightarrow \widetilde{\mathbb{H}}^{+}$, where $\widetilde{\mathbb{H}}^{+}$is the domain containing $\mathbb{H}^{+}$that is bounded by $E^{+}$and $\widetilde{\Psi}^{+}\left(\gamma_{1}^{-}\right){ }^{4}$

Interpolating functions. A sequence $\left\{z_{m}\right\}_{m=1}^{\infty} \subset \mathbb{H}^{+}$is called an $i n-$ terpolating sequence for $H^{\infty}\left(\mathbb{H}^{+}\right)$if, whenever $\left|w_{m}\right| \leq 1$, there exists a function $f \in H^{\infty}\left(\mathbb{H}^{+}\right)$such that

$$
f\left(z_{m}\right)=w_{m}, \quad m=1,2, \ldots
$$

When $\left\{z_{m}\right\}_{m=1}^{\infty}$ is an interpolating sequence, we call the finite bound

$$
\begin{aligned}
\mathcal{N}\left(\left\{z_{m}\right\}, \mathbb{H}^{+}\right)=\sup _{\left|w_{j}\right| \leq 1} \inf \left\{\|f\|: f \in H^{\infty}\left(\mathbb{H}^{+}\right)\right. \\
\left.\quad \text { and } \quad f\left(z_{m}\right)=w_{m}, m=1,2, \ldots\right\}
\end{aligned}
$$

the constant of interpolation. By a theorem of Carleson, $\left\{z_{m}\right\}_{m=1}^{\infty}$ is an interpolating sequence if and only if

$$
\delta_{\mathbb{H}^{+}}\left(\left\{z_{m}\right\}\right)=\inf _{n} \prod_{k, k \neq n}\left|\frac{z_{n}-z_{k}}{z_{n}-\overline{z_{k}}}\right|>0 ;
$$

furthermore, we have the relationship $1 / \delta_{\mathbb{H}^{+}} \leq \mathcal{N} \leq\left(1-\log \delta_{\mathbb{H}^{+}}\right) c / \delta_{\mathbb{H}^{+}}$, in which $c$ is some absolute constant. For a nice discussion on interpolating sequences and a proof of Carleson's interpolation theorem see [10, $\S \mathrm{VII}]$.

Fix $A=\frac{1-\beta_{1}}{1+3 \beta_{1}}$, and let $\left\{z_{m}\right\}_{m=1}^{\infty} \subset \mathbb{H}^{+}$be a sequence embedded in $\delta_{1}^{+}$satisfying

$$
\left|z_{n}-z_{m}\right| \geq A y_{m}, \quad n \neq m
$$

Since the sequence lies in a Carleson contour (by Lemma 2.2) and it is hyperbolically separated, we know that $\delta_{\mathbb{H}^{+}}\left(\left\{z_{m}\right\}\right)=C\left(A, \epsilon, \alpha_{M}\right)>$

$\overline{{ }^{4} \text { We are not identifying the crosscuts } \widetilde{\Psi}^{+}}\left(\gamma_{1}^{-}\right)$with the crosscuts $\delta_{1}^{-}$. 
0 (see $[\mathbf{1 0}, \S \mathrm{VII}]$ ). Carleson's interpolation theorem then implies that $\left\{z_{m}\right\}_{m=1}^{\infty}$ is an interpolating sequence for $\mathbb{H}^{+}$. It is also the case that $\left\{z_{m}\right\}_{m=1}^{\infty}$ is an interpolating sequence for the extended domain $\widetilde{\mathbb{H}}^{+}$. This follows from [11, Theorem IV.1], and applies in our case since $\widetilde{\mathbb{H}}^{+}$is a subset of a Denjoy domain. Alternatively, $\left\{z_{m}\right\}_{m=1}^{\infty}$ can be shown to be an interpolating sequence for $\widetilde{\mathbb{H}}^{+}$by a result of González and Nicolau [13]. From their result, it suffices to have $\delta_{\mathbb{H}^{+}}>0$ for the image of $\left\{\Phi^{+}\left(z_{m}\right)\right\}$ under the canonical quasi-conformal map that takes the domain $\left(\cup_{j} D_{j}\right) \cup \Omega^{+}$to the upper half plane. Since the quasi-conformal map is explicit, it is easy to verify; we omit the details. In any case, there exist interpolating functions for the domain $\widetilde{\mathbb{H}}^{+}$with a constant of interpolation $\mathcal{N}=\mathcal{N}\left(A, \alpha_{M}, \epsilon\right)$.

Working again in the upper half plane, let $B=\min \left\{A, \frac{1}{6 \mathcal{N}^{2}}\right\}$ and denote the region

$$
\mathcal{D}^{+}=\left\{z \in \mathbb{H}^{+}: \omega\left(z, \cup I_{j}^{+}, \mathbb{H}^{+}\right)>\beta_{1}, d(z)<B\right\}
$$

with $d(z)=y^{-1} \inf _{\zeta \in \delta_{1}^{+}}|z-\zeta|$. We chose $A$ so that by Harnack's inequality $\mathcal{D}^{+}$lies above $\delta_{2}^{+}$, that is

$$
\left.\omega\left(z, \cup I_{j}^{+}, \mathbb{H}^{+}\right)<\beta_{2}, \quad \text { for all } z \in \mathcal{D}^{+} \quad \text { (see Figure } 3\right)
$$

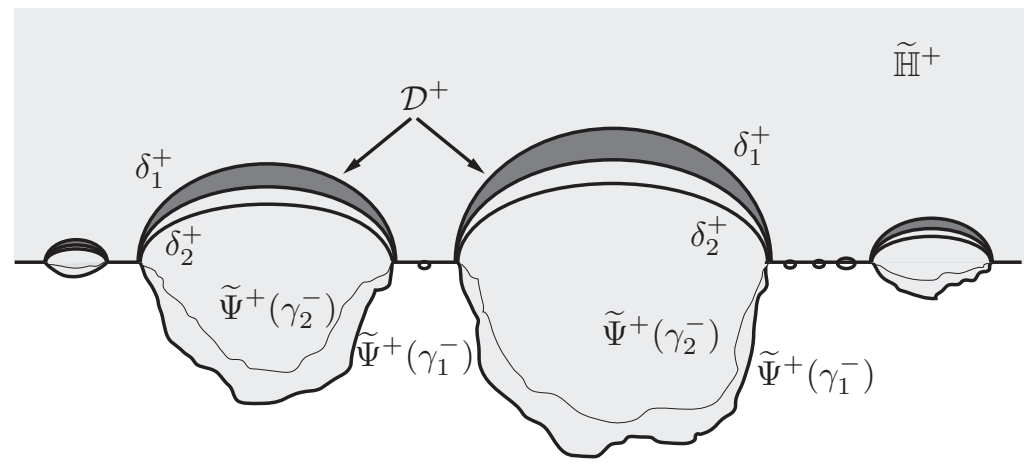

Figure 3 . The region $\mathcal{D}^{+}$lies between the curves $\delta_{1}^{+}$ and $\delta_{2}^{+}$and separates the two extended half planes. 
Now fix a sequence $\left\{z_{m}^{+}\right\} \subset \delta_{1}^{+}$satisfying

$$
\begin{aligned}
&\left|z_{n}^{+}-z_{m}^{+}\right| \geq B y_{m}^{+}, \quad n \neq m, \\
& \inf _{m} \frac{\left|z-z_{m}^{+}\right|}{y_{m}^{+}} \leq 3 B, \quad \text { for all } z \in \mathcal{D}^{+} .
\end{aligned}
$$

The existence of such a sequence follows by taking a maximal sequence satisfying (3.1). We now follow a standard argument as originated in [11, Lemma II.2] and as used in [14, Lemma III.2] to obtain a specific set of interpolation functions:

Lemma 3.1. There exists functions $\left\{h_{m}^{+}\right\}_{m=1}^{\infty} \subset H^{\infty}\left(\widetilde{\mathbb{H}}^{+}\right)$such that

$$
\begin{gathered}
h_{m}^{+}\left(z_{m}^{+}\right)=1, \\
\left\|h_{m}^{+}\right\|_{H^{\infty}} \leq \mathcal{N}^{2},
\end{gathered}
$$

and

$$
\sum_{m}\left|h_{m}^{+}(z)\right| \leq \mathcal{K}\left(A, \epsilon, \alpha_{M}\right) \quad z \in \widetilde{\mathbb{H}}^{+}
$$

Proof of Lemma 3.1: By a stopping time argument used to group $\left\{z_{m}^{+}\right\}$ into generations (see $\left[\mathbf{1 0}\right.$, p. 416]), we may split $\left\{z_{m}^{+}\right\}$into a finite union of disjoint subsequences $S_{k}, 1 \leq k \leq 2^{p}$, so that

$$
\inf \left\{\frac{\left|z_{j}^{+}-z_{l}^{+}\right|}{y_{l}^{+}}: z_{j}^{+}, z_{l}^{+} \in S_{k}, \quad j \neq l\right\} \geq A, \quad \text { for all } k \text {. }
$$

Since the points of $S_{k}$ are hyperbolically separated by $A$, our earlier discussion implies that each $S_{k}$ has a constant of interpolation less than $\mathcal{N}$.

Let us restrict our attention to a fixed subsequence $S_{k}$. If we assume that $S_{k}=\left\{z_{1}, z_{2}, \ldots, z_{n_{0}}\right\}$ is finite, then there exists $f_{j} \in H^{\infty}\left(\widetilde{\mathbb{H}}^{+}\right)$such that $\left\|f_{j}\right\|_{H^{\infty}} \leq \mathcal{N}$ and $f_{j}\left(z_{m}\right)=\omega^{m j}$, where $\omega=e^{2 \pi i / n_{0}}$. Moreover, if we define

$$
h_{m}^{+}(z)=\left(\frac{1}{n_{0}} \sum_{j=1}^{n_{0}} \omega^{-m j} f_{j}(z)\right)^{2}
$$


then $h_{m}^{+}\left(z_{j}\right)=\delta_{m, j}$ and

$$
\begin{aligned}
\sum_{m=1}^{n_{0}}\left|h_{m}^{+}(z)\right| & =n_{0}^{-2} \sum_{m=1}^{n_{0}} \sum_{j, l} \omega^{-m j} \omega^{m l} f_{j}(z) \overline{f_{l}}(z) \\
& =n_{0}^{-2} \sum_{j=1}^{n_{0}} n_{0}\left|f_{j}(z)\right|^{2} \leq \mathcal{N}^{2} .
\end{aligned}
$$

Therefore, by exhausting each $S_{k}$ and taking the normal limits, we have (3.3), (3.4), and (3.5) with $\mathcal{K}=\mathcal{N}^{2} 2^{p}$.

The technique used above of averaging interpolating functions is due to Varopoulos [28]. We made our choice of $\mathcal{N}^{2} B \leq 1 / 6$ specifically so that if we write $\mathcal{D}^{+}$as the disjoint union of sets $\mathcal{D}_{n}^{+} \subset\left\{z:\left|z-z_{n}^{+}\right| \leq\right.$ $\left.3 B y_{n}^{+}\right\}$, then with (3.4) and Schwarz Lemma

$$
\left|h_{n}^{+}(z)\right|>1 / 2 \quad \text { whenever } z \in \mathcal{D}_{n}^{+} .
$$

Since throughout this chapter we could change all plus signs to minus signs, we could likewise define our friends: $\widetilde{\Omega}^{-}, \widetilde{\Psi}^{-}, \widetilde{\mathbb{H}}^{-}, \mathcal{D}^{-},\left\{z_{m}^{-}\right\}_{m=1}^{\infty}$, and $\left\{h_{m}^{-}\right\}_{m=1}^{\infty}$.

\section{Iterative blending of corona solutions}

We now begin the process of "sewing" together corona solutions from the simply connected domains $\widetilde{\Omega}^{+}$and $\widetilde{\Omega}^{-}$. Let $\left\{g_{j}^{0}\right\}_{j=1}^{n}$ be an arbitrary corona solution set for $\widetilde{\Omega}^{+}$and let $\left\{g_{j}^{1}\right\}_{j=1}^{n}$ be an arbitrary corona solution set for $\widetilde{\Omega}^{-}$. These solution sets exist from Carleson's simply connected corona theorem; furthermore, there is a uniform bound for the sets:

$$
\left\|g_{j}^{0}\right\|_{H^{\infty}\left(\widetilde{\Omega}^{+}\right)} \leq N \quad \text { and } \quad\left\|g_{j}^{1}\right\|_{H^{\infty}\left(\widetilde{\Omega}^{-}\right)} \leq N, \quad j=1, \ldots, n .
$$

The bound, $N$, depends only on the corona constants: $N=N(\mu, \delta, n)$ $([\mathbf{1 0}, \S$ IIX $])$. In this chapter, we are going to create a special collection of solutions $\left\{g_{j}^{k}\right\}_{j=1}^{n} \subset H^{\infty}\left(\widetilde{\Omega}^{+}\right)$when $k$ is even, and $\left\{g_{j}^{k}\right\}_{j=1}^{n} \subset H^{\infty}\left(\widetilde{\Omega}^{-}\right)$ when $k$ is odd.

The first stitchings. The first sewing of the corona solutions will be across the region $\mathcal{D}^{+}$in $\widetilde{\mathbb{H}}^{+}$. Let us denote by $\omega(z)=\omega\left(z, \cup I_{j}^{+}, \mathbb{H}^{+}\right)$ as the harmonic measure for the open intervals $\bigcup_{j} I_{j}^{+}$in the upper half plane. Although $\omega(z)$ is not defined on the extended domain, we use the convention $\omega(\bar{z})=2-\omega\left(z, \cup I_{j}^{+}, \mathbb{H}^{+}\right.$) to extend $\omega$ to $\widetilde{\mathbb{H}}^{+}$. (When working 
in $\widetilde{\mathbb{H}}^{-}$, we will also be denoting with $\omega(z)$ and it should be clear from context.)

We can think of both $\left\{f_{j}(z)\right\}_{j=1}^{n}$ and $\left\{g_{j}^{0}(z)\right\}_{j=1}^{n}$ as being defined in $\widetilde{\mathbb{H}}^{+}$under the map $\widetilde{\Phi}^{+}(z)$, and we will not change our notation. On the other hand, when we write $\left\{g_{j}^{1}(z)\right\}_{j=1}^{n}$ we must remember that these functions are only defined in $\widetilde{\mathbb{H}}^{+}$between the curves $\left\{\widetilde{\Psi}^{+}\left(\gamma_{1}^{-}\right)\right\}$and $\left\{\delta_{1}^{+}\right\}$ (see Figure 4 ). Because of a calculation advantage, we have chosen not to sew on the $\widetilde{\Omega}^{+}$side where the corona data and solutions are originally defined, but instead work in the extended half planes where we have defined $\mathcal{D}^{+},\left\{z_{m}^{+}\right\}$, and $\left\{h_{m}^{+}(z)\right\}$.
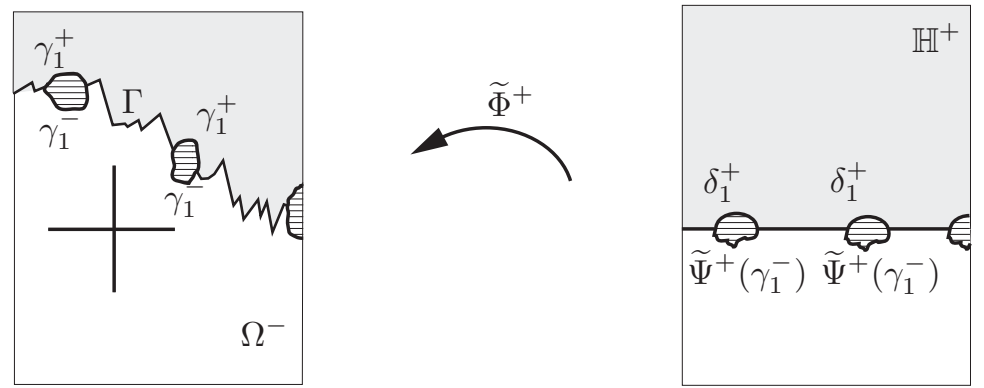

FIGURE 4. The checkered regions lie in the intersection of the domains for $\left\{g_{j}^{0}\right\}_{j=1}^{n}$ and $\left\{g_{j}^{1}\right\}_{j=1}^{n}$.

Let $\left\{\varphi^{+}(z), 1-\varphi^{+}(z)\right\}$ be a smooth partition of unity for $\widetilde{\mathbb{H}}^{+}$across $\mathcal{D}^{+}$ with $\varphi^{+}(z)=1$ when $\omega(z) \leq \beta_{1}$. By standard arguments, $\left|y_{n}^{+}\right|\left|\nabla \varphi^{+}(z)\right| \leq$ $C B^{-1}$ for all $z \in \mathcal{D}_{n}^{+}$, where $C$ is a positive constant. Using our partition, let us piece together the two families of corona solutions:

$$
G_{j}^{2}=g_{j}^{0} \varphi^{+}+g_{j}^{1}\left(1-\varphi^{+}\right) \quad j=1, \ldots, n .
$$

These smooth functions are a well defined solution set to the corona equation

$$
\sum f_{j}(z) G_{j}^{2}(z)=1
$$

for the region $\widetilde{\mathbb{H}}^{+}$, but they are not necessarily analytic. Therefore, we consider a technique of Hörmander [15] (and as used in [11] and [14]). We seek to find functions $\left\{a_{j, k}^{2}\right\} \subset L^{\infty}\left(\widetilde{\mathbb{H}}^{+}\right)$that solve (in the sense of distributions) the $\bar{\partial}$ equation

$$
\bar{\partial} a_{j, k}^{2}=G_{j}^{2} \bar{\partial} G_{k}^{2}
$$


Indeed, such functions provide the necessary cancelation to make the collection

$$
g_{j}^{2}=G_{j}^{2}+\sum_{k=1}^{n}\left(a_{j, k}^{2}-a_{k, j}^{2}\right) f_{k} \quad j=1, \ldots, n
$$

a solution set while simultaneously solving the equation $\bar{\partial} g_{j}^{2}=0$ in the sense of distributions. Then upon modifying each $a_{j, k}^{2}$ on a set of measure zero, Weyl's lemma will allow us to conclude that the collection $\left\{g_{j}^{2}\right\}_{j=1}^{n}$ is a bona fide corona solution set in $\widetilde{\mathbb{H}}^{+}$.

For our construction, we require not only that the functions $\left\{a_{j, k}^{2}\right\}$ are bounded, but also have an additional convergence factor. Fix $1>b_{1}>0$ (to be determined later) and denote by $\widetilde{\omega}(z)$ as the harmonic conjugate for $\omega(z)$. Consider the equation

$a_{j, k}^{2}(z)=\frac{1}{\pi} \sum_{l} \iint_{\mathcal{D}_{l}^{+}}\left(b_{1}^{\omega(z)-\omega(\zeta)+i(\widetilde{\omega}(z)-\widetilde{\omega}(\zeta))}\right) \frac{G_{j}^{2}(\zeta) \bar{\partial} G_{k}^{2}(\zeta)}{\zeta-z} \frac{h_{l}^{+}(z)}{h_{l}^{+}(\zeta)} d \zeta d \bar{\zeta}$

Formally $\bar{\partial} a_{j, k}^{2}=G_{j}^{2} \bar{\partial} G_{k}^{2}$, so we need to check the convergence of the sum

$$
\left|a_{j, k}^{2}(z)\right| \leq \frac{1}{\pi} \sum_{l} \iint_{\mathcal{D}_{l}^{+}}\left(b_{1}^{(\omega(z)-\omega(\zeta)}\right) \frac{\left|G_{j}^{2}(\zeta) \bar{\partial} G_{k}^{2}(\zeta)\right|}{|\zeta-z|} \frac{\left|h_{l}^{+}(z)\right|}{\left|h_{l}^{+}(\zeta)\right|} d \zeta d \bar{\zeta} .
$$

Using (3.6) and recalling $\omega(\zeta)<\beta_{2}$ when $z \in \mathcal{D}^{+}$,

$$
\begin{aligned}
\left|a_{j, k}^{2}(z)\right| & \leq \frac{2}{\pi} \sum_{l}\left|h_{l}^{+}(z)\right| \iint_{\mathcal{D}_{l}^{+}}\left(b_{1}^{\omega(z)-\omega(\zeta)}\right) \frac{\left|G_{j}^{2}(\zeta) \bar{\partial} G_{k}^{2}(\zeta)\right|}{|\zeta-z|} d \zeta d \bar{\zeta} \\
& \leq \frac{2}{\pi} \sum_{l}\left|h_{l}^{+}(z)\right|\left(b_{1}^{\left(\omega(z)-\beta_{2}\right)}\right) \iint_{\mathcal{D}_{l}^{+}} \frac{\left|G_{j}^{2}(\zeta) \bar{\partial} G_{k}^{2}(\zeta)\right|}{|\zeta-z|} d \zeta d \bar{\zeta} \\
& \leq \frac{2}{\pi} \sum_{l}\left|h_{l}^{+}(z)\right|\left(b_{1}^{\left(\omega(z)-\beta_{2}\right)}\right) \iint_{\mathcal{D}_{l}^{+}} \frac{\left|G_{j}^{2}(\zeta)\right|\left|g_{k}^{1}(\zeta)-g_{k}^{0}(\zeta)\right|\left|\nabla \varphi^{+}(\zeta)\right|}{|\zeta-z|} d \zeta d \bar{\zeta}
\end{aligned}
$$

Before we show the above is a convergent sum, we would like to identify some key numbers that will appear in the iterative process. Using the notation $\|\cdot\|_{\mathcal{D}^{+}}$and $\|\cdot\|_{\mathcal{D}^{-}}$for the supremum of the modulus in the 
region $\mathcal{D}^{+}$and $\mathcal{D}^{-}$respectively, let us label

$$
\begin{aligned}
& x_{m}= \begin{cases}\max _{k}\left\|g_{k}^{m}-g_{k}^{m-1}\right\|_{\mathcal{D}^{+}} & \text {when } m \text { is odd, } \\
\max _{k}\left\|g_{k}^{m}-g_{k}^{m-1}\right\|_{\mathcal{D}^{-}} & \text {when } m \text { is even, }\end{cases} \\
&9 p t] y_{m}= \begin{cases}\max _{k}\left\|G_{k}^{m+1}\right\|_{\mathcal{D}^{+}} & \text {when } m \text { is odd, } \\
\max _{k}\left\|G_{k}^{m+1}\right\|_{\mathcal{D}^{-}} & \text {when } m \text { is even. }\end{cases}
\end{aligned}
$$

So that in our context,

$$
\begin{aligned}
\left|a_{j, k}^{2}(z)\right| & \leq \frac{2}{\pi} \sum_{l}\left|h_{l}^{+}(z)\right| b_{1}^{\left(\omega(z)-\beta_{2}\right)} x_{1} y_{1} \iint_{\mathcal{D}_{l}^{+}} \frac{\left|\nabla \varphi^{+}(\zeta)\right|}{|\zeta-z|} d \zeta d \bar{\zeta} \\
& \leq(12 C) \sum_{l}\left|h_{l}^{+}(z)\right| b_{1}^{\left(\omega(z)-\beta_{2}\right)} x_{1} y_{1}
\end{aligned}
$$

and by using (3.5) we reduce the inequality to

$$
\left|a_{j, k}^{2}(z)\right| \leq(12 C) \mathcal{K} b_{1}^{\left(\omega(z)-\beta_{2}\right)} x_{1} y_{1}
$$

We conclude that $\left\{a_{j, k}^{2}\right\} \subset L^{\infty}\left(\widetilde{\mathbb{H}}^{+}\right)$as it is easy to verify $x_{1}$ and $y_{1}$ are bounded with $N$. Moreover, if we apply these bounds to the relationship (4.1), then we get the bounded equation

$$
\left|g_{j}^{2}(z)-G_{j}^{2}(z)\right| \leq K b_{1}^{\left(\omega(z)-\beta_{2}\right)} x_{1} y_{1}, \quad \text { for all } z \in \widetilde{\mathbb{H}}^{+},
$$

where $K$ is an absolute constant which depends only upon the corona constants $(\mu, \delta$, and $n)$, the geometric considerations $\left(\epsilon\right.$ and $\left.\alpha_{M}\right)$, and our choice of $A$. Lastly, under the map $\Phi^{+}(z)$ we can regard the newly constructed $\left\{g_{j}^{2}\right\}_{j=1}^{n}$ and $\left\{G_{j}^{2}\right\}_{j=1}^{n}$ as being functions defined on $\widetilde{\Omega}^{+}$.

The subsequent stitchings. In the same fashion that we used to construct the relationship (4.1), we could construct the third generation of solutions, $\left\{g_{j}^{3}\right\}_{j=1}^{n}$ and $\left\{G_{j}^{3}\right\}_{j=1}^{n}$, by stitching the newly formed $\left\{g_{j}^{2}\right\}_{j=1}^{n}$ to $\left\{g_{j}^{1}\right\}_{j=1}^{n}$ across the region $\mathcal{D}^{-}$in $\widetilde{\mathbb{H}}^{-}$. As soon as the third generation of solutions are constructed, we repeat the process, just as we did in the first stitchings, to obtain the fourth generation of solutions, $\left\{g_{j}^{4}\right\}_{j=1}^{n}$ and $\left\{G_{j}^{4}\right\}_{j=1}^{n}$, by stitching $\left\{g_{j}^{3}\right\}_{j=1}^{n}$ to $\left\{g_{j}^{2}\right\}_{j=1}^{n}$ across $\mathcal{D}^{+}$in $\widetilde{\mathbb{H}}^{+}$. Iterating this procedure with the sequences $\left\{b_{m}\right\}_{m=1}^{\infty},\left\{x_{m}\right\}_{m=1}^{\infty}$, and $\left\{y_{m}\right\}_{m=1}^{\infty}$, 
we deduce the analogues of (4.1) and (4.2):

$$
\begin{array}{ll}
g_{j}^{m}=G_{j}^{m}+\sum_{k=1}\left(a_{j, k}^{m}-a_{k, j}^{m}\right) f_{k} & m=2,3, \ldots, \\
\left|g_{j}^{m+1}(z)-G_{j}^{m+1}(z)\right| \leq K b_{m}^{\left(\omega(z)-\beta_{2}\right)} x_{m} y_{m} & m=1,2, \ldots
\end{array}
$$

Since we will be referring to (4.4) many times from here, we consider some variations. Each variation is customized to the location of the variable $z$. Recall,

$$
\begin{aligned}
& G_{j}^{m+1}(z)= \begin{cases}g_{j}^{m}(z) & \text { when } m \text { is odd, } z \in \widetilde{\mathbb{H}}^{+}, \text {and } z \text { lies below } \delta_{2}^{+}, \\
g_{j}^{m-1}(z) & \text { when } m \text { is odd, } z \in \widetilde{\mathbb{H}}^{+}, \text {and } z \text { lies above } \delta_{1}^{+},\end{cases} \\
& G_{j}^{m+1}(z)= \begin{cases}g_{j}^{m}(z) & \text { when } m \text { is even, } z \in \widetilde{\mathbb{H}}^{-}, \text {and } z \text { lies above } \delta_{2}^{-}, \\
g_{j}^{m-1}(z) & \text { when } m \text { is even, } z \in \widetilde{\mathbb{H}}^{-}, \text {and } z \text { lies below } \delta_{1}^{-} .\end{cases}
\end{aligned}
$$

The phrases " $z$ lies below $\delta_{2}^{+}$" and " $z$ lies above $\delta_{1}^{+}$" when referring to $z \in$ $\widetilde{\mathbb{H}}^{+}$formally means $\omega(z) \geq \beta_{2}$ and $\omega(z) \leq \beta_{1}$ respectively. Similarly when $z \in \widetilde{\mathbb{H}}^{-}$, " $z$ lies above $\delta_{2}^{-}$" and " $z$ lies below $\delta_{1}^{+}$" means $\omega(z) \geq \beta_{2}$ and $\omega(z) \leq \beta_{1}$. Immediately, we obtain two variations:

(4.4a) $\left|g_{j}^{m+1}(z)-g_{j}^{m-1}(z)\right| \leq K b_{m}^{-\beta_{2}} x_{m} y_{m}$ $m$ odd, $z \in \widetilde{\mathbb{H}}^{+}$, and $z$ lies above $\delta_{1}^{+}$,

(4.4b) $\left|g_{j}^{m+1}(z)-g_{j}^{m-1}(z)\right| \leq K b_{m}^{-\beta_{2}} x_{m} y_{m}$

$$
m \text { even, } z \in \widetilde{\mathbb{H}}^{-} \text {, and } z \text { lies below } \delta_{1}^{-} \text {. }
$$

We observe that in region

$$
\begin{aligned}
V^{+} & =\widetilde{\mathbb{H}}^{+} \backslash \overline{\mathbb{H}^{+}} \\
& =\left\{z \in \widetilde{\mathbb{H}}^{+}: z \text { lies strictly below } \bigcup_{j} I_{j}^{+} \text {and strictly above } \widetilde{\Psi}^{+}\left(\gamma_{1}^{-}\right)\right\}
\end{aligned}
$$

we have a lower bound on harmonic measure: $\omega(z)>1$. With this observation and (4.4), we note

$$
\left|g_{j}^{m+1}(z)-g_{j}^{m}(z)\right| \leq K b_{m}^{\left(1-\beta_{2}\right)} x_{m} y_{m} \quad \text { when } m \text { is odd and } z \in V^{+} .
$$

Under the map $\widetilde{\Psi}^{-} \circ \widetilde{\Phi}^{+}$, we transfer the preceding relationship to the extended lower half plane (and repeat the construction for $m$ even with 
the region $\left.V^{-}\right)$:

$$
\begin{aligned}
\left|g_{j}^{m+1}(z)-g_{j}^{m}(z)\right| \leq & K b_{m}^{\left(1-\beta_{2}\right)} x_{m} y_{m} \\
& \text { when } m \text { is odd, } z \in \widetilde{\Psi}^{-}\left(\widetilde{\Phi}^{+}\left(V^{+}\right)\right) \subset \widetilde{\mathbb{H}}^{-},
\end{aligned}
$$

(4.4d) $\left|g_{j}^{m+1}(z)-g_{j}^{m}(z)\right| \leq K b_{m}^{\left(1-\beta_{2}\right)} x_{m} y_{m}$ when $m$ is even, $z \in \widetilde{\Psi}^{+}\left(\widetilde{\Phi}^{-}\left(V^{-}\right)\right) \subset \widetilde{\mathbb{H}}^{+}$.
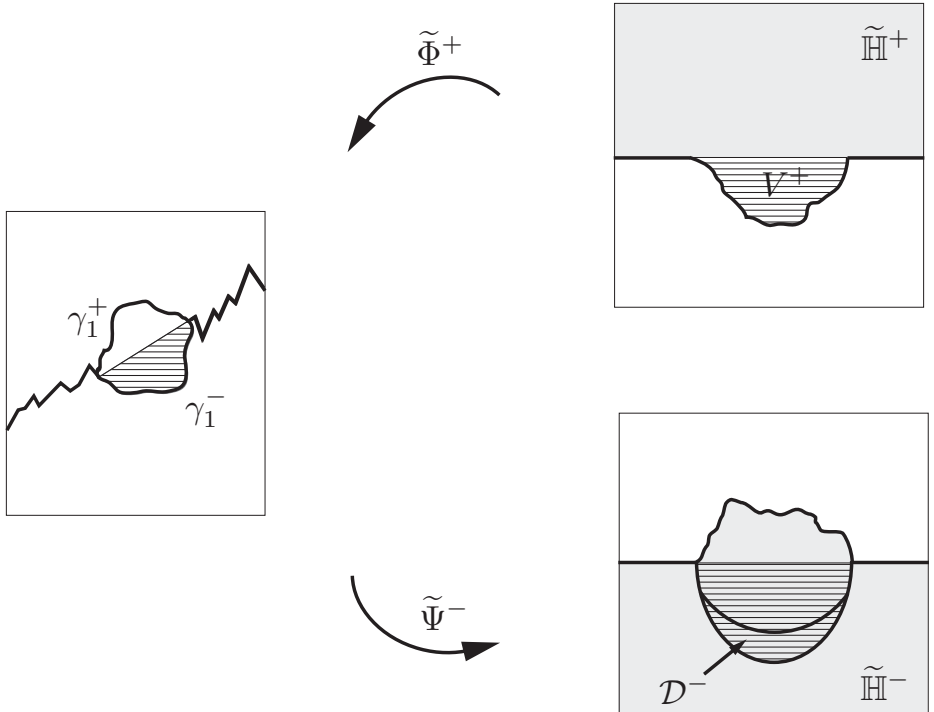

Figure 5. The region $V+$ under the map $\widetilde{\Psi}^{-} \circ \widetilde{\Phi}^{+}$.

From the latter two variations and observing that $\mathcal{D}^{+} \subset \widetilde{\Psi}^{+}\left(\widetilde{\Phi}^{-}\left(V^{-}\right)\right)$ and $\mathcal{D}^{-} \subset \widetilde{\Psi}^{-}\left(\widetilde{\Phi}^{+}\left(V^{+}\right)\right)$, we deduce our first recursive relationship:

$$
x_{1} \leq 2 N
$$

Next, let us deduce a recursive relationship for $\left\{y_{m}\right\}_{m=1}^{\infty}$. It is easy to verify $y_{1} \leq N$. Now suppose $m$ is odd, then $y_{m+2}=\max _{k}\left\|G_{k}^{m+3}\right\|_{\mathcal{D}^{+}}$ $=\max _{k}\left\|g_{k}^{m+2}\left(\varphi^{+}\right)+g_{k}^{m+1}\left(1-\varphi^{+}\right)\right\|_{\mathcal{D}^{+}}$. 
Let us take a look at the bounds for the functions in the above equality. Recall, $\omega(z)>\beta_{1}$ when $z \in \mathcal{D}^{+}$so that (4.4) reduces to

$$
\left|g_{k}^{m+1}(z)\right| \leq\left|G_{k}^{m+1}(z)\right|+K b_{m}^{\left(\beta_{1}-\beta_{2}\right)} x_{m} y_{m} .
$$

In addition, (4.4d) and the previous inequality imply

$$
\begin{aligned}
\left|g_{k}^{m+2}(z)\right| & \leq\left|g_{k}^{m+1}(z)\right|+K b_{m+1}^{\left(1-\beta_{2}\right)} x_{m+1} y_{m+1} \\
& \leq\left|G_{k}^{m+1}(z)\right|+K b_{m}^{\left(\beta_{1}-\beta_{2}\right)} x_{m} y_{m}+K b_{m+1}^{\left(1-\beta_{2}\right)} x_{m+1} y_{m+1} .
\end{aligned}
$$

Since the bound for $\left|g_{k}^{m+2}(z)\right|$ is greater than the bound for $\left|g_{k}^{m+1}(z)\right|$, we deduce that

$$
\begin{aligned}
y_{m+2} & =\max _{k}\left\|g_{k}^{m+2}\left(\varphi^{+}\right)+g_{k}^{m+1}\left(1-\varphi^{+}\right)\right\|_{\mathcal{D}^{+}} \\
& \leq \max _{k}\left\|\left|G_{k}^{m+1}(z)\right|+K b_{m}^{\left(\beta_{1}-\beta_{2}\right)} x_{m} y_{m}+K b_{m+1}^{\left(1-\beta_{2}\right)} x_{m+1} y_{m+1}\right\|_{\mathcal{D}^{+}} \\
& \leq \max _{k}\left\|G_{k}^{m+1}(z)\right\|_{\mathcal{D}^{+}}+K b_{m}^{\left(\beta_{1}-\beta_{2}\right)} x_{m} y_{m}+K b_{m+1}^{\left(1-\beta_{2}\right)} x_{m+1} y_{m+1} \\
& =y_{m}+K b_{m}^{\left(\beta_{1}-\beta_{2}\right)} x_{m} y_{m}+K b_{m+1}^{\left(1-\beta_{2}\right)} x_{m+1} y_{m+1} .
\end{aligned}
$$

We could repeat verbatim the case where $m$ is even, and for the case of $y_{2}$ with the bound $\left\|g_{j}^{1}\right\|_{\infty} \leq N$, so that we obtain the second recursive relationship:

$$
y_{1} \leq N, \quad y_{2} \leq N+K b_{1}^{\left(1-\beta_{2}\right)} x_{1} y_{1},
$$

$$
y_{m+2} \leq y_{m}+K b_{m}^{\left(\beta_{1}-\beta_{2}\right)} x_{m} y_{m}+K b_{m+1}^{\left(1-\beta_{2}\right)} x_{m+1} y_{m+1} \quad m=1,2, \ldots
$$

The last two terms in this expression arise from the error in stitching over two generations. It will be our goal to show that these errors are summable.

\section{Convergence of $x_{m}$}

In this chapter we select the factors $\left\{b_{m}\right\}_{m=1}^{\infty}$ so that $\left\{x_{m}\right\}_{m=1}^{\infty} \in \ell^{1}$ and $\left\{y_{m}\right\}_{m=1}^{\infty} \in \ell^{\infty}$ simultaneously:

Lemma 5.1. For positive constants $N, K, \beta_{1}$, and $\beta_{2}$, with $\beta_{1}, \beta_{2}<$ 1 and $\left(1-\beta_{1}\right) /\left(1-\beta_{2}\right)=2$, there exists a sequence of positive real numbers $\left\{b_{m}\right\}_{m=1}^{\infty}$ with

$$
0<\inf _{m}\left\{b_{m}\right\} \quad \text { and } \quad b_{m}<1
$$


such that for any pair of positive sequences $\left\{x_{m}\right\}_{m=1}^{\infty}$ and $\left\{y_{m}\right\}_{m=1}^{\infty}$ that satisfies the difference equations

(R1) $x_{m+1} \leq K b_{m}^{\left(1-\beta_{2}\right)} x_{m} y_{m} \quad m=1,2, \ldots$

(R2) $y_{m+2} \leq y_{m}+K b_{m}^{\left(\beta_{1}-\beta_{2}\right)} x_{m} y_{m}+K b_{m+1}^{\left(1-\beta_{2}\right)} x_{m+1} y_{m+1} \quad m=1,2, \ldots$

with initial data $x_{1} \leq 2 N, y_{1} \leq N$, and $y_{2} \leq N+K b_{1}^{\left(1-\beta_{2}\right)} x_{1} y_{1}$, will also satisfy

$$
\sum_{m=1}^{\infty} x_{m}<C_{1}<\infty \text { and } \sup _{m}\left\{y_{m}\right\}<C_{2}<\infty .
$$

Proof of Lemma 5.1: Since the pair of sequences $\left(\left\{x_{m}\right\}_{m=1}^{\infty}\right.$ and $\left.\left\{y_{m}\right\}_{m=1}^{\infty}\right)$ that have equality holding in the initial data and equality holding in (R1) and (R2) dominate all admissible pairs, it suffices to solve for $\left\{b_{m}\right\}_{m=1}^{\infty}$ for this particular pair. In addition, without loss of generality, we may assume that $y_{1}, y_{2} \geq 1$. Now, fix $1>r>0$ (to be determined later) and take $b_{m}$ so that

$$
x_{m+1} \stackrel{(\mathrm{R} 1)}{=} K b_{m}^{\left(1-\beta_{2}\right)} x_{m} y_{m}=r^{m} \quad m=1,2, \ldots
$$

Next, we substitute the right hand side of the above equation to reduce (R2),

$$
y_{m+2}=y_{m}+b_{m}^{\left(\beta_{1}-1\right)} r^{m}+r^{m+1} \quad m=1,2, \ldots
$$

When $m \geq 2$, we can solve for $b_{m}$ in terms of $r$ and $y_{m}$ by looking at successive generations of (5.1). Specifically, the right hand side of (5.1) at the $m^{\text {th }}$ generation is

$$
K b_{m}^{\left(1-\beta_{2}\right)} x_{m} y_{m}=r^{m},
$$

and by substituting the left hand side of (5.1) for $x_{m}$ makes

$$
K b_{m}^{\left(1-\beta_{2}\right)} r^{m-1} y_{m}=r^{m},
$$

so that

$$
K b_{m}^{\left(1-\beta_{2}\right)} y_{m}=r
$$

If we raise both sides of the above equality to the power $\left(\frac{\beta_{1}-1}{1-\beta_{2}}\right)=-2$, then

$$
b_{m}^{\left(\beta_{1}-1\right)}=\left(\frac{K y_{m}}{r}\right)^{2}
$$


For the case where $m=1$ we can repeat the preceding, but with $x_{1}=2 \mathrm{~N}$ to obtain

$$
b_{1}^{\left(\beta_{1}-1\right)}=\left(\frac{K y_{1}(2 N)}{r}\right)^{2} .
$$

If we substitute these relations for $b_{m}$ and $b_{1}$ into (5.2), then we have the ordinary difference equation:

$$
y_{1}=N, \quad y_{2}=N+r, \quad y_{3}=y_{1}+K^{2} y_{1}^{2}(2 N)^{2} r^{-1}+r^{2},
$$

$$
y_{m+2}=y_{m}+K^{2} y_{m}^{2} r^{m-2}+r^{m+1} \quad m=2,3, \ldots
$$

To get bounds for $y_{m}$, we look at the difference

$$
\begin{aligned}
\frac{1}{y_{m}}-\frac{1}{y_{m+2}}=\frac{y_{m+2}-y_{m}}{y_{m} y_{m+2}} & =K^{2}\left(\frac{y_{m}}{y_{m+2}}\right) r^{m-2}+\frac{r^{m+1}}{y_{m} y_{m+2}} \\
& \leq K^{2} r^{m-2}+r^{m+1} \quad m=2,3, \ldots
\end{aligned}
$$

The last inequality holds since $1 \leq \cdots \leq y_{m} \leq y_{m+2}$. By telescoping the differences, starting with $y_{4}$ and $y_{5}$ for $m$ even and $m$ odd respectively,

$$
\begin{aligned}
& \frac{1}{y_{4}}-\frac{1}{y_{m+2}} \leq K^{2} \sum_{\substack{j=4, j \text { even }}}^{m} r^{j-2}+\sum_{\substack{j=4, j \text { even }}}^{m} r^{j+1}=\mathcal{O}\left(r^{2}\right) \quad m \text { even, } m>2, \\
& \frac{1}{y_{5}}-\frac{1}{y_{m+2}} \leq K^{2} \sum_{\substack{j=5, j \text { odd }}}^{m} r^{j-2}+\sum_{\substack{j=5, j \text { odd }}}^{m} r^{j+1}=\mathcal{O}\left(r^{3}\right) \quad m \text { odd, } m>3 .
\end{aligned}
$$

We recall, $y_{1}=N, y_{2}=N+r$; and by using $(5.4 \mathrm{a})$ and $(5.4 \mathrm{~b}), y_{3}=$ $\mathcal{O}\left(r^{-1}\right), y_{4}=\mathcal{O}(1)$, and $y_{5}=\mathcal{O}\left(r^{-1}\right)$. So that for $r$ sufficiently small, $0<C(r)<\frac{1}{y_{4}}-\left(K^{2} \sum_{\substack{j=4, j \text { even }}}^{m} r^{j-2}+\sum_{\substack{j=4, j \text { even }}}^{m} r^{j+1}\right) \leq \frac{1}{y_{m+2}} \quad m$ even, $m>2$, $0<C(r)<\frac{1}{y_{5}}-\left(K^{2} \sum_{\substack{j=5, j \text { odd }}}^{m} r^{j-2}+\sum_{\substack{j=5, j \text { odd }}}^{m} r^{j+1}\right) \leq \frac{1}{y_{m+2}} \quad m$ odd, $m>3$. 
Fix such an $r$ small enough so that the above inequalities holds and make sure $r>r_{0}>0$ so that,

$$
\sup _{m}\left\{y_{m}\right\}<C_{2}\left(r_{0}\right) \text { and } \sum_{m=1}^{\infty} x_{m} \leq 2 N+\sum_{m=2}^{\infty} r^{m-1}<C_{1},
$$

while

$$
1>b_{1}=\left(\frac{r}{K y_{1}(2 N)}\right)^{\frac{2}{1-\beta_{1}}} \geq\left(\frac{r_{0}}{K C_{2}(2 N)}\right)^{\frac{2}{1-\beta_{1}}}
$$

and

$$
1>b_{m}=\left(\frac{r}{K y_{m}}\right)^{\frac{2}{1-\beta_{1}}} \geq\left(\frac{r_{0}}{K C_{2}}\right)^{\frac{2}{1-\beta_{1}}} m=2,3, \ldots
$$

We conclude that $\inf _{m}\left\{b_{m}\right\}>0$, and thus $\left\{b_{m}\right\}_{m=1}^{\infty}$ is our desired sequence.

\section{Proof of Theorem $\mathbf{1 . 1}$}

With the sequence $\left\{b_{m}\right\}_{m=1}^{\infty}$ following from Lemma 5.1, we now show:

$$
\sup _{m \text { even }}\left\|g_{j}^{m}\right\|_{H^{\infty}\left(\widetilde{\mathbb{H}}^{+}\right)} \leq C<\infty \quad j=1, \ldots, n,
$$

and

$$
\sup _{\operatorname{modd}}\left\|g_{j}^{m}\right\|_{H^{\infty}\left(\widetilde{\mathbb{H}}^{-}\right)} \leq C<\infty \quad j=1, \ldots, n,
$$

where $C$ is some absolute constant depending only upon $\epsilon_{0}, \alpha$, and $A$. We begin by looking at $\left\|g_{j}^{m}\right\|_{H^{\infty}\left(\widetilde{\mathbb{H}}^{+}\right)}$in the extended upper half plane. Fix $z \in \widetilde{\mathbb{H}}^{+}$and $m$ odd. From (4.4) and variation (4.4a) for the regions $\left\{\omega(z) \leq \beta_{1}\right\},\left\{\beta_{1}<\omega(z) \leq \beta_{2}\right\}$, and $\left\{\beta_{2}<\omega(z)\right\}$ respectively, we have

(6.1) $\left|g_{j}^{m+1}(z)-g_{j}^{m-1}(z)\right| \leq K b_{m}^{-\beta_{2}} x_{m} y_{m} \quad$ when $z$ lies above $\delta_{1}^{+}$,

(6.2) $\left|g_{j}^{m+1}(z)\right| \leq\left|G_{j}^{m+1}(z)\right|+K b_{m}^{\left(\beta_{1}-\beta_{2}\right)} x_{m} y_{m} \quad$ when $z$ lies below $\delta_{1}^{+}$ and above $\delta_{2}^{+}$

(6.3) $\left|g_{j}^{m+1}(z)-g_{j}^{m}(z)\right| \leq K x_{m} y_{m}$ when $z$ lies below $\delta_{2}^{+}$.

Let us treat each region as its separate own special case. 
Case i) $z$ lies above $\delta_{1}^{+}$.

From the first relationship (6.1), if we telescope the differences over the even generations of $\left\{g_{j}^{m}\right\}$, then

$\left|g_{j}^{m+1}(z)\right| \leq N+\sum_{\substack{k=1, k \text { odd }}}^{\infty} K b_{k}^{-\beta_{2}} x_{k} y_{k} \leq N+K\left(\frac{K C_{2}}{r_{0}}\right)^{\frac{2 \beta_{2}}{1-\beta_{1}}} \sum_{\substack{k=1, k \text { odd }}}^{\infty} x_{k} y_{k} \leq C<\infty$,

since $\sup _{k}\left\{y_{k}\right\}<C_{2}, \sum_{k} x_{k}<C_{1}$, and $\inf _{k}\left\{b_{k}\right\} \geq\left(\frac{r_{0}}{K C_{2}}\right)^{\frac{2}{1-\beta_{1}}}$ from Lemma 5.1.

Case ii) $z$ lies below $\delta_{1}^{+}$and above $\delta_{2}^{+}$.

For this case and the next we need some estimates similar to the ones we obtained when we derived the second recursive relation, (R2). Recall the relationship we have from (4.4d) in this region,

$$
\left|g_{j}^{1}(z)-g_{j}^{0}(z)\right| \leq 2 N,
$$

and

$$
\left|g_{j}^{m}(z)-g_{j}^{m-1}(z)\right| \leq K b_{m-1}^{\left(1-\beta_{2}\right)} x_{m-1} y_{m-1} \quad m \text { odd, } m>1 .
$$

As $G_{j}^{m+1}$ is an average of the two functions in the above,

$$
\left|G_{j}^{m+1}(z)\right|=\left|g_{j}^{m}(z)\left(1-\varphi^{+}(z)\right)+g_{j}^{m-1}(z) \varphi^{+}(z)\right|,
$$

we can create two inequalities depending on whether we choose to bound $g_{j}^{m+1}$ or $g_{j}^{m}$ :

1) $\left|G_{j}^{m+1}(z)\right| \leq\left|g_{j}^{m-1}(z)\right|+K b_{m-1}^{\left(1-\beta_{2}\right)} x_{m-1} y_{m-1}$, m odd, $m>1$.

2) $\left|G_{j}^{m+1}(z)\right| \leq\left|g_{j}^{m}(z)\right|+K b_{m-1}^{\left(1-\beta_{2}\right)} x_{m-1} y_{m-1}$, m odd, $m>1$.

If we choose the first inequality, (6.2) reduces to

(6.4a) $\left|g_{j}^{2}(z)\right| \leq 2 N+K b_{1}^{\left(\beta_{1}-\beta_{2}\right)} x_{1} y_{1}$,

and

$$
\left|g_{j}^{m+1}(z)\right| \leq\left|g_{j}^{m-1}(z)\right|+K b_{m}^{\left(\beta_{1}-\beta_{2}\right)} x_{m} y_{m}+K b_{m-1}^{\left(1-\beta_{2}\right)} x_{m-1} y_{m-1},
$$

and if we choose the second inequality (6.2) reduces to

(6.4b) $\left|g_{j}^{2}(z)\right| \leq 2 N+K b_{1}^{\left(\beta_{1}-\beta_{2}\right)} x_{1} y_{1}$,

and

$$
\left|g_{j}^{m+1}(z)\right| \leq\left|g_{j}^{m}(z)\right|+K b_{m}^{\left(\beta_{1}-\beta_{2}\right)} x_{m} y_{m}+K b_{m-1}^{\left(1-\beta_{2}\right)} x_{m-1} y_{m-1}
$$


Now for case ii), (6.4a) unfolds to

$$
\begin{aligned}
\left|g_{j}^{m+1}(z)\right| & \leq 2 N+\sum_{\substack{k=1, k \text { odd }}}^{\infty} K b_{k}^{\left(\beta_{1}-\beta_{2}\right)} x_{k} y_{k}+\sum_{\substack{k=2, k \text { even }}}^{\infty} K b_{k}^{\left(1-\beta_{2}\right)} x_{k} y_{k} \\
& \leq 2 N+K\left(\frac{K C_{2}}{r_{0}}\right)^{\frac{2\left(\beta_{2}-\beta_{1}\right)}{1-\beta_{1}}} \sum_{k=1}^{\infty} x_{k} y_{k} \leq C<\infty
\end{aligned}
$$

Case iii) $z$ lies below $\delta_{2}^{+}$.

By the conformal map $\widetilde{\Psi}^{+} \circ \widetilde{\Phi}^{-}$, we have the relationships for the $m-1^{\text {th }}$ ( $m$ odd, $\left.m>1\right)$ generation of $(6.3)$ and $(6.4 \mathrm{~b})$ respectively:

$$
\begin{array}{r}
\left|g_{j}^{m}(z)-g_{j}^{m-1}(z)\right| \leq K x_{m-1} y_{m-1} \quad \text { when z lies above } \widetilde{\Psi}^{+}\left(\gamma_{2}^{-}\right), \\
\left|g_{j}^{m}(z)\right| \leq\left|g_{j}^{m-1}(z)\right|+K b_{m-1}^{\left(\beta_{1}-\beta_{2}\right)} x_{m-1} y_{m-1}+K b_{m-2}^{\left(1-\beta_{2}\right)} x_{m-2} y_{m-2} \\
\text { when z lies below } \widetilde{\Psi}^{+}\left(\gamma_{2}^{-}\right) \text {and above } \widetilde{\Psi}^{+}\left(\gamma_{1}^{-}\right) .
\end{array}
$$

In the first event, we combine with (6.3) to get

$$
\left|g_{j}^{m+1}(z)\right| \leq\left|g_{j}^{m-1}(z)\right|+K x_{m} y_{m}+K x_{m-1} y_{m-1},
$$

and thus

$$
\left|g_{j}^{m+1}(z)\right| \leq N+\sum_{k=1}^{\infty} K x_{k} y_{k} \leq C<\infty .
$$

In the second event, we combine with (6.3) to get

$$
\begin{aligned}
\left|g_{j}^{m+1}(z)\right| \leq\left|g_{j}^{m-1}(z)\right|+K x_{m} y_{m} & +K b_{m-1}^{\left(\beta_{1}-\beta_{2}\right)} x_{m-1} y_{m-1} \\
& +K b_{m-2}^{\left(1-\beta_{2}\right)} x_{m-2} y_{m-2},
\end{aligned}
$$

and thus

$$
\left|g_{j}^{m+1}(z)\right| \leq N+2 K \sum_{k=1} b_{k}^{\left(\beta_{1}-\beta_{1}\right)} x_{k} y_{k} \leq C<\infty
$$

We conclude that $\left\|g_{j}^{m}\right\|_{H^{\infty}\left(\widetilde{\mathbb{H}}^{+}\right)} \leq C$ for all even $m$, since we have bounded these functions over the whole domain $\widetilde{\mathbb{H}}^{+}$. Similarly, (with a lower bound) we could repeat the above procedure to conclude that $\left\|g_{j}^{m}\right\|_{H^{\infty}\left(\widetilde{\mathbb{H}}^{-}\right)} \leq C$ for all $m$ odd over the domain $\widetilde{\mathbb{H}}^{-}$. Not only are $\left\{g_{j}^{m}\right\}_{\{m \text {, even }\}}$ and $\left\{g_{j}^{m}\right\}_{\{m \text {, odd }\}}$ uniformly bounded in their respective domains, for each $j$, but also their difference has a shrinking bound in the intersection of $\widetilde{\Omega}^{+}$and $\widetilde{\Omega}^{-}$. We demonstrate this by showing that 
the sequences are uniformly Cauchy in $\Gamma \backslash E_{0}=\bigcup_{j} F_{j}$ in the following sense:

Let $n>m \geq 0$ and let $z \in \Gamma \backslash E_{0}$, then with (4.4) and (5.1),

$$
\left|g_{j}^{n}(z)-g_{j}^{m}(z)\right| \leq \sum_{k=m}^{n-1}\left|g_{j}^{k}(z)-g_{j}^{k+1}(z)\right| \leq \sum_{k=m}^{n-1} K b_{k}^{\left(1-\beta_{2}\right)} x_{k} y_{k}=\sum_{k=m}^{n-1} r^{k}
$$

Now, let $\left\{g_{j}^{+}\right\}_{j=1}^{n}$ be the normal limit of $\left\{g_{j}^{m}\right\}_{m=0}^{\infty} \subset H^{\infty}\left(\widetilde{\Omega}^{+}\right)$for $m$ even, and let $\left\{g_{j}^{-}\right\}_{j=1}^{n}$ be the normal limit of $\left\{g_{j}^{m}\right\}_{m=1}^{\infty} \subset H^{\infty}\left(\widetilde{\Omega}^{-}\right)$ for $m$ odd. As point-wise limits

$$
\begin{array}{ll}
g_{1}^{+}(z) f_{1}(z)+g_{2}^{+}(z) f_{2}(z)+\cdots+g_{n}^{+}(z) f_{n}(z)=1 & z \in \widetilde{\Omega}^{+}, \\
g_{1}^{-}(z) f_{1}(z)+g_{2}^{-}(z) f_{2}(z)+\cdots+g_{n}^{-}(z) f_{n}(z)=1 & z \in \widetilde{\Omega}^{-} .
\end{array}
$$

Moreover, (6.5) implies that $g_{k}^{+}(z)=g_{k}^{-}(z)$ for all $z \in \Gamma \backslash E_{0}$. Therefore, we can merge the two solutions together across $\Gamma \backslash E_{0}$, and obtain corona solutions on all of $\Omega$.

For our proof, the homogeneous condition was critical. Without it, we would not have been able to bound the crosscuts $\gamma_{1}^{+}$and $\gamma_{1}^{-}$into the disjoint diamonds, leaving the extended domains as multiply connected. A proof for the non-homogeneous case still eludes the author. One might hope to avoid this obstacle by directly applying the results of the nonhomogeneous cases, (e.g., the Denjoy domains).

The present work is part of the author's Ph.D. dissertation. Most of all, the author would like to express his genuine gratitude to his thesis advisor, John Garnett, for countless hours of insightful conversations and guidance throughout the past couple years. The author is truly indebted for his support.

\section{References}

[1] N. L. Alling, A proof of the corona conjecture for finite open Riemann surfaces, Bull. Amer. Math. Soc. 70 (1964), 110-12.

[2] N. L. Alling, Extensions of meromorphic function rings over noncompact Riemann surfaces. I, Math. Z. 89 (1965), 273-299.

[3] M. Behrens, The corona conjecture for a class of infinitely connected domains, Bull. Amer. Math. Soc. 76 (1970), 387-391.

[4] L. Carleson, Interpolations by bounded analytic functions and the corona problem, Ann. of Math. (2) 76 (1962), 547-559.

[5] L. Carleson, On $H^{\infty}$ in multiply connected domains, in: "Conference on harmonic analysis in honor of Antoni Zygmund", Vol. I, II 
(Chicago, Ill., 1981), Wadsworth Math. Ser., Wadsworth, Belmont, CA, 1983, pp. 349-372.

[6] C. J. EArle And A. Marden, Projections to automorphic functions, Proc. Amer. Math. Soc. 19 (1968), 274-278.

[7] F. Forelli, Bounded holomorphic functions and projections, Illinois J. Math. 10 (1966), 367-380.

[8] T. W. Gamelin, Wolff's proof of the corona theorem, Israel J. Math. 37(1-2) (1980), 113-119.

[9] T. W. Gamelin, Localization of the corona problem, Pacific J. Math. 34 (1970), 73-81.

[10] J. B. Garnett, "Bounded analytic functions", Revised first edition, Graduate Texts in Mathematics 236, Springer, New York, 2007.

[11] J. B. Garnett and P. W. Jones, The corona theorem for Denjoy domains, Acta Math. 155(1-2) (1985), 27-40.

[12] M. J. GonzÁlez, Uniformly perfect sets, Green's function, and fundamental domains, Rev. Mat. Iberoamericana 8(2) (1992), 239-269.

[13] M. J. GonzÁlez And A. Nicolau, Quasiconformal mappings preserving interpolating sequences, Ann. Acad. Sci. Fenn. Math. 23(2) (1998), 283-290.

[14] J. HANDY, The corona theorem on the complement of certain square Cantor sets, J. Anal. Math. 108 (2009), 1-18.

[15] L. Hörmander, Generators for some rings of analytic functions, Bull. Amer. Math. Soc. 73 (1967), 943-949.

[16] P. W. Jones, Carleson measures and the Fefferman-Stein decomposition of $\operatorname{BMO}(\mathbf{R})$, Ann. of Math. (2) 111(1) (1980), 197-208.

[17] P. W. Jones, $L^{\infty}$ estimates for the $\bar{\partial}$ problem in a half-plane, Acta Math. 150(1-2) (1983), 137-152.

[18] P. W. Jones, Some problems in complex analysis, in: "The Bieberbach conjecture" (West Lafayette, Ind., 1985), Math. Surveys Monogr. 21, Amer. Math. Soc., Providence, RI, 1986, pp. 105-108.

[19] P. W. Jones and D. E. Marshall, Critical points of Green's function, harmonic measure, and the corona problem, Ark. Mat. 23(2) (1985), 281-314.

[20] C. E. Kenig, Weighted $H^{p}$ spaces on Lipschitz domains, Amer. J. Math. 102(1) (1980), 129-163.

[21] B. Muckenhoupt, The equivalence of two conditions for weight functions, Studia Math. 49 (1973/74), 101-106.

[22] Ch. Pommerenke, Uniformly perfect sets and the Poincaré metric, Arch. Math. (Basel) 32(2) (1979), 192-199. 
[23] Z. Slodkowski, On bounded analytic functions in finitely connected domains, Trans. Amer. Math. Soc. 300(2) (1987), 721-736.

[24] E. L. Stout, Two theorems concerning functions holomorphic on multiply connected domains, Bull. Amer. Math. Soc. 69 (1963), 527-530.

[25] E. L. Stout, Bounded holomorphic functions on finite Reimann surfaces, Trans. Amer. Math. Soc. 120 (1965), 255-285.

[26] E. L. Stout, On some algebras of analytic functions on finite open Riemann surfaces, Math. Z. 92 (1966), 366-379; Corrections to: "On some algebras of analytic functions on finite Riemann surfaces", Math. Z. 95 (1967), 403-404.

[27] M. TsuJI, "Potential theory in modern function theory", Maruzen Co., Ltd., Tokyo, 1959.

[28] Th. VAropoulos, Ensembles pics et ensembles d'interpolation pour les algèbres uniformes, C. R. Acad. Sci. Paris Sér. A-B $\mathbf{2 7 2}$ (1971), A866-A867.

Department of Mathematics

University of California

Los Angeles, CA 90095-1555

USA

E-mail address: bnewdelm@math.ucla.edu

Primera versió rebuda el 4 de desembre de 2009 ,

darrera versió rebuda el 9 de setembre de 2010. 